\title{
Improved theoretical description of Mueller-Navelet jets at LHC
}

\author{
Dimitri Colferai* \\ Dimitri Colferai (Dipartimento di Fisica, Università di Firenze and INFN Firenze) \\ E-mail: colferai@fi.infn.it
}

\author{
Alessandro Niccoli \\ (Dipartimento di Fisica, Università di Firenze) \\ E-mail: niccoliefi.infn.it

\section{Federico Deganutti} \\ (Dipartimento di Fisica, Università di Firenze) \\ E-mail: fedeganutti@gmail.com
}

\begin{abstract}
We present a method for improving the phenomenological description of Mueller-Navelet jets at LHC, which is based on matching the BFKL resummation with fixed order calculations. We will also point out the need of a consistent treatment of jets between experimental measurements and theoretical descriptions. We hope as well to motivate an extensive analysis of MN jets at LHC in run 2.
\end{abstract}

QCD Evolution 2016

May 30-June 03, 2016

National Institute for Subatomic Physics (Nikhef), Amsterdam

\footnotetext{
* Speaker.
} 


\section{Introduction and outline}

The framework of this work is the study of the high-energy behaviour of QCD in the Regge limit, namely at high energy. Under these conditions, standard perturbation theory (PT) at fixed order fails, and new phenomena are expected to arise, like power-like behaviour of the amplitudes and cross sections. Since the pioneering studies in the 70's [1], it has become clear that a perturbative description of QCD processes is possible, provided infinite classes of diagrams are resummed to all orders in the strong coupling, so as to take into account all the contributions of the form $\left(\alpha_{s} \log s\right)^{n}$.

With the advent of high-energy colliders, HERA, TEV, LHC, there have been various attempts to check the validity of the BFKL approach. At hadron colliders, one of the most suitable observable for such a check was proposed by Mueller and Navelet (MN) [2], and consists of measuring the production rate of inclusive events characterized by two jets with large rapidity separation.

Even if BFKL and MN jets are old ideas, after many years of studies they still represent an open problem, which deserves further investigations, especially now that LHC is delivering so high energetic collisions. In fact, after the experimental analysis by CMS, the comparison with BFKL and also with MC predictions turned out not to be satisfactory, thus calling for improvements that some groups have implemented.

Our proposal of improvement is based on two observations:

- firstly, we point out that, so far, theoretical descriptions are not fully consistent with experimental analysis, regarding the jet definition and data selection; we shall show how to modify the theoretical description in a way consistent with experimental analysis;

- after such modification, improvements are still needed. We present our method based on matching the resummed BFKL description with the fixed next-to-leading order (NLO) calculations, and then we show some preliminary results.

\section{Mueller-Navelet jets within the BFKL approach}

MN jets are inclusive events with a pair of jets having large rapidity separation $Y \equiv \mid y_{1}-$ $y_{2} \mid \gg 1$ and comparable transverse momenta $p_{T 1} \sim p_{T 2}$. Anything can be emitted in between the jets. The fact of having comparable hard scales (jet $p_{T}$ 's) limits the logarithms of collinear type $\left(\log p_{T 1} / p_{T 2}\right)$, while the big separation in rapidity enhances the $\operatorname{large} \log \left(s / p_{T}{ }^{2}\right) \simeq Y$.

The theoretical BFKL description of MN jets is based on a double factorization formula (fig. 1)

$$
\frac{\mathrm{d} \sigma(s)}{\mathrm{d} J_{1} \mathrm{~d} J_{2}}=\sum_{a, b} \int_{0}^{1} \mathrm{~d} x_{1} \mathrm{~d} x_{2} \int \mathrm{d}^{2} k_{1} \mathrm{~d}^{2} k_{2} f_{a}\left(x_{1}\right) V_{a}\left(x_{1}, k_{1} ; J_{1}\right) G\left(x_{1} x_{2} s, k_{1}, k_{2}\right) V_{b}\left(x_{2}, k_{2} ; J_{2}\right) f_{b}\left(x_{2}\right),
$$

where $J_{i}=\left(y_{i}, p_{T i}, \phi_{i}\right)$ denotes jet variables. On the external side, we have the usual collinear factorization which expresses the hadronic differential cross section as a convolution of partonic distribution functions (PDF) and a hard-scattering partonic cross section. In turn, the partonic cross section at high energy can be expressed by means of a $k_{T}$-dependent factorization formula.

In the middle there is the so-called gluon Green function (GGF) $G$, which represents the sum of all ladder diagrams with reggeized gluon exchanges, and obeys the BFKL equation, whose integral 
kernel $K$ represents one rung of the ladder and is computable in perturbation theory. The impact factors $V$ describe the coupling of the Reggeized gluon with the external particles. In this case of incoming parton and outgoing jet, the impact factors are called jet vertices.

In the leading-logarithmic (LL) approximation, the jet vertex is trivial, being simply identified with the scattered parton, since the LL kinematics is characterized by large rapidity gaps among emitted particles.

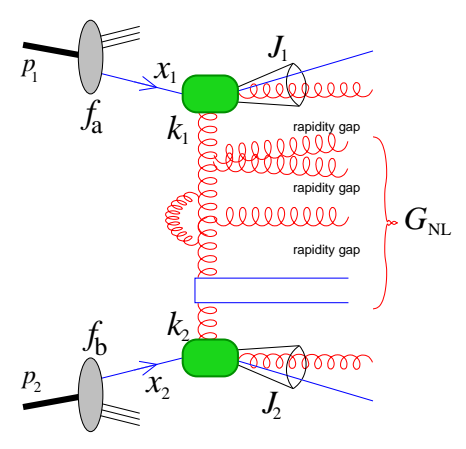

Figure 1: Schematic representation of the factorization formula for MN jets.

At next-to-leading (NLL) level, Bartels, Vacca and myself [3] proved a factorization formula with the same structure. Both the GGF and jet vertices receives NLL correction, and in this case the jets can have a non-trivial structure (fig. 1). In particular they depend on the jet resolution $R$ and on the jet algorithm.

With LHC these ideas can be tested, and few years ago, Schwennsen, Szymanowski Wallon and myself made a careful study of MN jets for the design energy at $14 \mathrm{TeV}$ [4].

\section{CMS measurements of MN jets}

From the experimental side, in 2012 CMS published an analysis of MN jets from data collected during the $7 \mathrm{TeV}$ run [5]. They have analysed the distribution of the azimuthal angle $\phi$ between the two jets. It is zero when they are emitted back-to-back, as in leading order (LO) PT, while it can be different from zero when additional radiation is present. Since BFKL predicts an amount of radiation that is different (typically larger) than fixed order calculations, one could expect to reveal the signal of BFKL dynamics from decorrelation measurements.

In addition, the CMS collaboration measured the average value of the cosine of that angle and higher angular moments, as well as their ratios. The advantage of these observables relies in the partial cancellation of various sistematic errors like PDF and scale uncertainties.

Fig. 2 shows the distribution $\frac{1}{\sigma} \frac{\mathrm{d} \sigma}{\mathrm{d} \phi}$ of the azimuthal angle between the MN jets, whose rapidity $y_{j}$ can be as large as 4.7 units. The data (black points) are compared with various MC predictions. We see that some $\mathrm{MC}$ are close to data but only in limited ranges of $\phi$. At higher rapidity differences the situation is similar, with larger errors on data. The overall description is not good.

Figs. 3 show the $Y$-dependence of the angular moments

$$
\langle\cos (m \phi)\rangle=\frac{C_{m}(Y)}{C_{0}(Y)} \equiv \frac{\int \mathrm{d} \phi \frac{\mathrm{d}^{2} \sigma}{\mathrm{d} \phi \mathrm{d} Y} \cos (m \phi)}{\mathrm{d} \sigma / \mathrm{d} Y}
$$



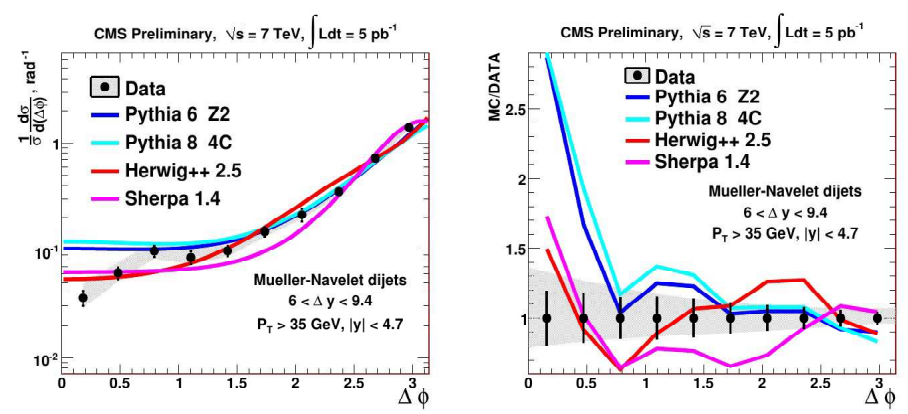

Figure 2: CMS measurement of the azimuthal distribution of MN jets, compared with MC predictions.
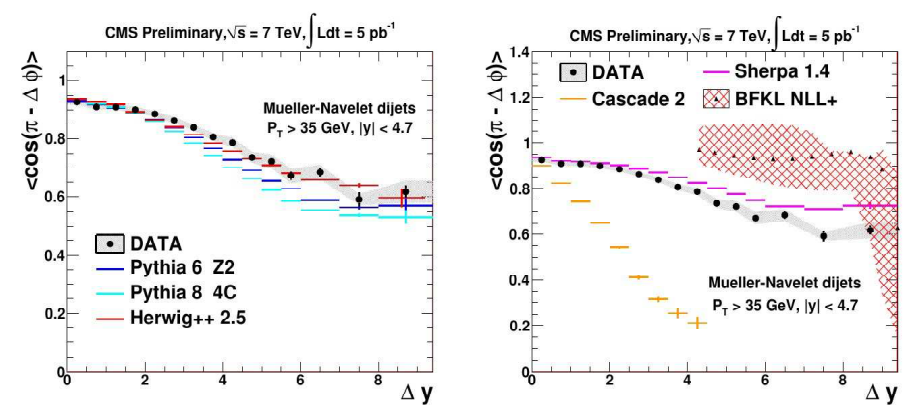

Figure 3: CMS measurements of the ratio $C_{1} / C_{0}=\langle\cos (\phi)\rangle$ versus $Y$, compared with MC and BFKL predictions. Here $\Delta y$ corresponds to $Y$ in the text, while $\pi-\Delta \phi$ with $\phi$.

In this case data were compared with both $\mathrm{MC}$ and BFKL predictions. Looking at $C_{1} / C_{0}$, as expected, there is more radiation with increasing $Y$ and thus more decorrelation. However BFKL shows less decorrelation, contrary to expectations. Some MC are close to data, but others are not and NLL BFKL is definitely off, despite the rather large uncertainty band due essentially to scale variations. The situation is similar for higher angular moments like $C_{2} / C_{0}$.

Surprisingly, the ratio $C_{2} / C_{1}$ is in perfect agreement with BFKL, while MCs don't agree with data.

In practice, neither NLL BFKL nor fixed order MC give a satisfactory description of data yet; furthermore NLL BFKL suffers from large scale uncertainties of the order of $10 \% \div 15 \%$.

\section{MN jets with BLM scale fixing}

In order to improve the BFKL description, various optimization procedure were used. Ducloué, Szymanowski and Wallon (DSW) proposed to tame the large scale dependence of BFKL by fixing the renormalization scale according to the BLM procedure [6]. This prescription provides a good description of data for the distribution of the azimuth between jets, where data and theory are compatible within errors. A good agreement is also found for the ratios of angular coefficients $C_{m} / C_{n}$. However, the price to pay for such agreement is the use of a renormalization scale $\mu_{R}^{2} \sim(10 \div 20)^{2} p_{T 1} p_{T 2}$ which is much larger than the physical scales of the process.

Other methods were proposed to improve the BFKL description of data. DSW again try to take into account energy momentum conservation (which is only approximate within the BFKL 
approach) by using an effective rapidity as suggested long ago by Del Duca and Schmidt [7].

CIMP considered instead various representations of the NLL cross section by fixing energy scales with PMS, FAC, BLM [8].

The underlying idea is to effectively include higher order corrections which are missing in NLL BFKL. However, as shown by these authors, the obtained results are quite different for the various prescriptions, and eventually only BLM does a fairly good job.

To include higher order corrections is probably needed, but to some extent it is an arbitrary procedure. On the other hand, there is a rigorous improvement that can be done with the actual knowledge of QCD, that is matching NLL BFKL with perturbative NLO (and possibly NNLO) calculations, and this is the path that we follow.

\section{Modification of jet vertices for consistency with CMS}

Actually, during our recent studies, we have realized that the definition of jet vertices proposed by Bartes, Vacca and myself in 2002 [3], checked in 2011 by Caporale, Ivanov, Murdaca, Papa and Perri [9], and used until now in the description of $\mathrm{MN}$ jets, is not equivalent to the event selection adopted in the measurement of the CMS collaboration.

In fact, in the experimental analysis:

- particles are first clustered into jets;

- then one considers only jets with $p_{T}$ above some threshold, in that case $35 \mathrm{GeV}$;

- finally, the tagged jets (i.e., the MN jets) are the farthest in rapidity.

This is not exactly what was prescribed in the definition of jet vertices in NLL BFKL approximation. The procedure for determining the vertex in, say, the forward region (positive rapidity) goes as follows:

- The first step is to choose a set of numbers for the jet variables $J=\left(p_{T}, \phi, y\right)$

- Next, for each event, the two partons with largest rapidities are subject to the clustering algorithm; the cross section receives contribution only if there is a jet with the specified variables.

- if such jet is formed by both partons, the probability of this event contributes to the NLL jet vertex;

- instead, if the jet contains only one parton, we look at the other parton:

$*$ if it is found in the fragmentation region of incoming hadron, this contribution is collinear singular and we consider it as a PDF correction;

* if it is a gluon in central region, this event contributes to the GGF

$*$ in all other cases, the events contribute to the jet vertex correction.

The important point to notice is that, in this last case, the parton outside the tagged jet can be hard $\left(p_{T}>35 \mathrm{GeV}\right)$ and can also be emitted at rapidity $y>y_{J}$. At the hadronic level, this parton 
gives rise to a jet. This means that we are allowing jets with rapidity greater than that of the MN jet, in contrast to the experimental selection of events described in the previous slide.

In other words, if the two partons with largest rapidity are not clustered into a single jet, and both of them have $p_{T}>35 \mathrm{GeV}$, this event contributes twice to the MN jet cross section: one time with $J=p_{1}$, the other time with $J=p_{2}$; On the contrary, in the CMS experimental analysis, this event contributes only once, the jet being identified always with the parton having largest rapidity.

Conceptually, this is a big difference. However, we have to remember that, since the MN jets have a large rapidity distance, it is rather unlikely to have the emission of another parton at even larger rapidities (i.e., outside such rapidity interval). For this reason, we don't expect big differences between the two procedures.
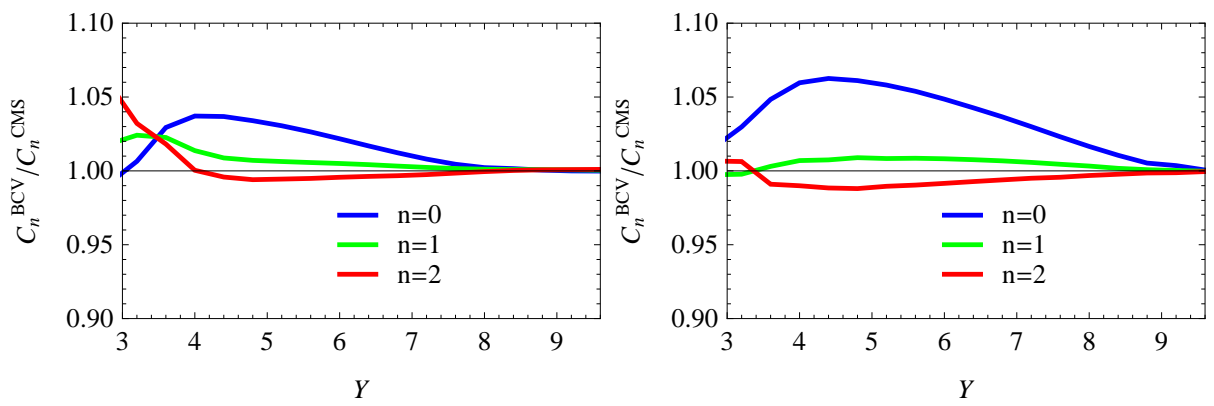

Figure 4: Ratios of angular coefficients using the original definition of jet vertices [3] with the modified definition which is consistent with the CMS data selection. Left: $\sqrt{s}=7 \mathrm{TeV}$; right: $\sqrt{s}=13 \mathrm{TeV}$.

In fig. 4 we compare the NLL BFKL prediction having the original NLL jet vertices (red) with the modified one that prevents the emission of a parton with $p_{T}>35 \mathrm{GeV}$ and $y>y_{J}$ (blue). We find differences of the order of $4 \%$ at intermediate rapidities $(Y \sim 3 \div 5)$ and much smaller at larger rapidities. However, the relative difference almost doubles at $13 \mathrm{TeV}$, and cannot be neglected.

Let me just mention that it could be possible to modify the experimental analysis of $\mathrm{MN}$ jets in order to comply with our original proposal of NLL jet vertices, but we think that in this case it is better to modify the theoretical prescription by requiring the absence of jets with $p_{T}>35 \mathrm{GeV}$ and $y>y_{J}$.

\section{Matching BFKL with fixed order perturbation theory}

Finally, we present the matching procedure. We believe that exploiting all the actual knowledge of PT theory should produce more reliable results and improve the description of data, with reduced scale uncertainties. Our hope is also to improve the estimate of cross section, and not just the azimuthal decorrelation. In this case, we think that fixed NLO and probably NNLO corrections must be fully taken into account.

The matching procedure is rather standard, though tricky at some point, as we shall see. In practice, we add to BFKL the full perturbative NLO result, and then subtract the $\mathscr{O}\left(\alpha_{s}^{3}\right)$ part already included in BFKL, in order to avoid double counting.

We will show results for cross section and angular coefficients. The implementation is still work in progess, therefore we can show only preliminary results of central values, without error estimate yet. However, an important lesson can be drawn for future phenomenological analysis. 
The structure of the matched cross section is given by

$$
\begin{aligned}
\frac{\mathrm{d} \sigma(s)}{\mathrm{d} J_{1} \mathrm{~d} J_{2}}= & \sum_{a, b} \int_{0}^{1} \mathrm{~d} x_{1} \mathrm{~d} x_{2} f_{a}\left(x_{1}\right) f_{b}\left(x_{2}\right)\{ \\
& \int \mathrm{d}^{2} k_{1} \mathrm{~d}^{2} k_{2}\left[V_{a}^{(0+1)}\left(x_{1}, k_{1} ; J_{1}\right) G_{\mathrm{NLL}}\left(x_{1} x_{2} s, k_{1}, k_{2}\right) V_{b}^{(0+1)}\left(x_{2}, k_{2} ; J_{2}\right)\right] \\
& +\frac{\mathrm{d} \hat{\sigma}^{(N L O)}\left(x_{1}, x_{2}\right)}{\mathrm{d} J_{1} \mathrm{~d} J_{2}} \\
& -\int \mathrm{d}^{2} k_{1} \mathrm{~d}^{2} k_{2}\left[V_{a}^{(0)}\left(x_{1}, k_{1} ; J_{1}\right) \delta^{2}\left(k_{1}-k_{2}\right) V_{b}^{(0)}\left(x_{2}, k_{2} ; J_{2}\right)\right] \\
& -\int \mathrm{d}^{2} k_{1} \mathrm{~d}^{2} k_{2}\left[V_{a}^{(1)}\left(x_{1}, k_{1} ; J_{1}\right) \delta^{2}\left(k_{1}-k_{2}\right) V_{b}^{(0)}\left(x_{2}, k_{2} ; J_{2}\right)\right] \\
& -\int \mathrm{d}^{2} k_{1} \mathrm{~d}^{2} k_{2}\left[V_{a}^{(0)}\left(x_{1}, k_{1} ; J_{1}\right) \delta^{2}\left(k_{1}-k_{2}\right) V_{b}^{(1)}\left(x_{2}, k_{2} ; J_{2}\right)\right] \\
& \left.-\int \mathrm{d}^{2} k_{1} \mathrm{~d}^{2} k_{2}\left[V_{a}^{(0)}\left(x_{1}, k_{1} ; J_{1}\right) \alpha_{s} \log \frac{x_{1} x_{2} s}{s_{0}} K_{0}\left(k_{1}, k_{2}\right) V_{b}^{(0)}\left(x_{2}, k_{2} ; J_{2}\right)\right]\right\}
\end{aligned}
$$

Besides the two parton densities, we have the BFKL expression for the hadronic cross section with NLL jet vertices and GGF (second line), to which add the full NLO PT contribution, (third line). In order to avoid double counting, we have to subtract the LO (fourth line) and 1-loop corrections of the BFKL expansion (last three lines).

Let us start with the computation of the differential cross section with respect to $Y$ ( fig. 5). Here the center-of-mass energy is $\sqrt{s}=7 \mathrm{TeV}$ and we impose symmetric cuts $p_{T i}>35 \mathrm{GeV}$ on the two jet transverse momenta, as in the CMS analysis. The green line is the BFKL prediction. The fixed order result, represented in red, turns out to be negative! Furthermore, after several days of computing time, $\mathrm{MC}$ errors are still large, due to the slow convergence of the integrand. However, also the double-counting subtraction (purple) is negative. Since the difference between red and purple is moderate, the matched cross section (blue) receives a moderate correction and remains positive. An analogue behaviour is shared by the first azimuthal coefficient $C_{1}$, where the
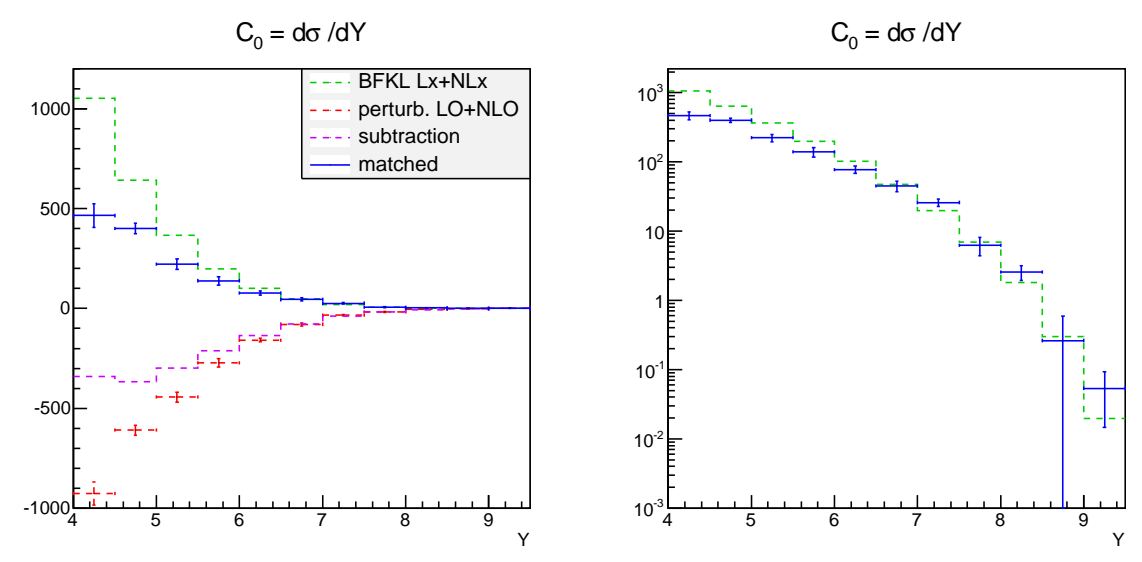

Figure 5: BFKL, fixed order, subtraction and matched cross section versus $Y$, for symmetric cuts $p_{T 1,2}>$ $35 \mathrm{GeV}$. Left: linear scale; right: logarithmic scale.

cross section is weighted with the cosine of the azimuthal angle between the jets. The matching 
procedure provides moderate but definitely sizeable corrections, in particular at intermediate values of $Y$.

Let me briefly discuss the issue of negative cross section and instability of jet cross section with symmetric cuts. It is well known that jet cross sections at NLO are very sensitive to the asymmetry parameter $\Delta \equiv p_{T 1}-p_{T 2}$ which measures the imbalance between the $p_{T}$ cuts on the two jets. Despite the fact that NLO cross sections are finite for all values of $\Delta$, they are affected by a $\Delta \log (\Delta)$ singularity of collinear origin, which provides an infinite derivative at $\Delta=0$, i.e., with symmetric jet cuts, and even negative cross sections for small $\Delta$, as we have seen.

However, and analogous singularity occurs in the subtraction term, as can be seen in the expansion of the BFKL cross section. It turns out that in the matching procedure such singular terms cancel out to a large extent, so that the matching is quite safe. Actually, this problem could be avoided by using asymmetric cuts on the jet momenta, but this introduces an unpleasant asymmetry and enhances collinear contributions.

Fortunately, there is another way to avoid (or at least to reduce) this problem while keeping symmetry between the two jets, that is to impose a cut on the sum of the jet transverse momenta, e.g., $\frac{1}{2}\left(p_{T 1}+p_{T 2}\right)>35 \mathrm{GeV}$. In this case, also the fixed-order cross section is well behaved, and the whole procedure is more stable than the previous one (fig. 6). The same is true for the $C_{1}$ angular coefficient. For this reason, we strongly suggests experimentalists to perform $\mathrm{MN}$ jets analysis
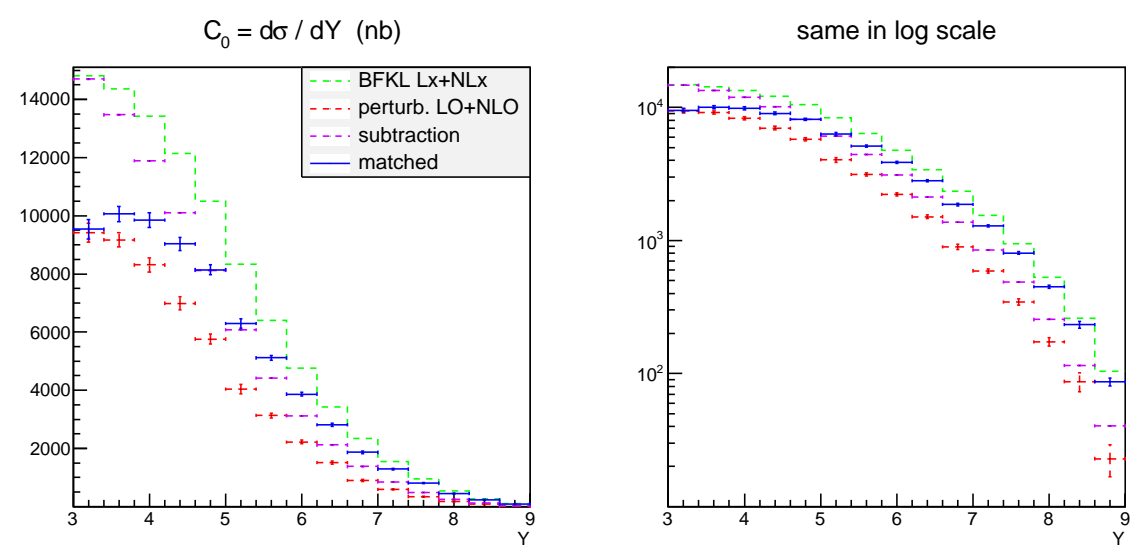

Figure 6: Same as fig. 5 but for symmetric cut $\frac{1}{2}\left(p_{T 1}+p_{T 2}\right)>35 \mathrm{GeV}$.

with a cut on the average of the jet transverse momenta, as already done in other jet analysis. This allows smaller theoretical uncertainties so that MN jets become a better tool for finding evidence of BFKL dynamics which, at present energies, is still competing with fixed order contributions, even at LHC.

To conclude, $\mathrm{MN}$ jets are a good observable for demonstrating the presence of BFKL dynamics at high energy, with yet open questions to be answered. We propose an improved theoretical description of MNJ, based on matching BFKL resummed calculations with fixed-order one. We have computed various observables and we will soon provide a full analysis with estimates of theoretical uncertainties.

We think that a precise experimental analysis from the $13 \mathrm{TeV}$ run would be very valuable for the study of QCD at high energies. In this respect, we strongly suggest experimentalists of LHC to 
carry out such analysis by imposing different cuts on the jet transverse momenta, for instance a cut on the average transverse momentum of jets.

\section{References}

[1] V. S. Fadin, E. A. Kuraev and L. N. Lipatov, "On the Pomeranchuk Singularity in Asymptotically Free Theories," Phys. Lett. B 60 (1975) 50; E. A. Kuraev, L. N. Lipatov and V. S. Fadin, "Multi - Reggeon Processes in the Yang-Mills Theory," Sov. Phys. JETP 44 (1976) 443 [Zh. Eksp. Teor. Fiz. 71 (1976) 840] [Erratum-ibid. 45 (1977) 199]; E. A. Kuraev, L. N. Lipatov and V. S. Fadin, "The Pomeranchuk Singularity in Nonabelian Gauge Theories," Sov. Phys. JETP 45 (1977) 199 [Zh. Eksp. Teor. Fiz. 72 (1977) 377]; I. I. Balitsky and L. N. Lipatov, "The Pomeranchuk Singularity in Quantum Chromodynamics," Sov. J. Nucl. Phys. 28 (1978) 822 [Yad. Fiz. 28 (1978) 1597].

[2] A. H. Mueller and H. Navelet, "An Inclusive Minijet Cross-Section and the Bare Pomeron in QCD," Nucl. Phys. B 282 (1987) 727.

[3] J. Bartels, D. Colferai and G. P. Vacca, "The NLO jet vertex for Mueller-Navelet and forward jets: The Quark part," Eur. Phys. J. C 24 (2002) 83 [hep-ph/0112283]; J. Bartels, D. Colferai and G. P. Vacca, "The NLO jet vertex for Mueller-Navelet and forward jets: The Gluon part," Eur. Phys. J. C 29 (2003) 235 [hep-ph/0206290].

[4] D. Colferai, F. Schwennsen, L. Szymanowski and S. Wallon, "Mueller Navelet jets at LHC - complete NLL BFKL calculation,” JHEP 1012 (2010) 026 [arXiv:1002.1365 [hep-ph]].

[5] CMS Collaboration [CMS Collaboration], "Azimuthal angle decorrelations of jets widely separated in rapidity in pp collisions at $\sqrt{s}=7 \mathrm{TeV}$," CMS-PAS-FSQ-12-002.

[6] B. Ducloué, L. Szymanowski and S. Wallon, "Confronting Mueller-Navelet jets in NLL BFKL with LHC experiments at 7 TeV," JHEP 1305 (2013) 096 [arXiv:1302.7012 [hep-ph]].

[7] B. Ducloué, L. Szymanowski and S. Wallon, "Violation of energy-momentum conservation in Mueller-Navelet jets production,” Phys. Lett. B 738 (2014) 311 doi:10.1016/j.physletb.2014.09.025 [arXiv:1407.6593 [hep-ph]].

[8] F. Caporale, D. Y. Ivanov, B. Murdaca and A. Papa, "Mueller-Navelet jets in next-to-leading order BFKL: theory versus experiment," Eur. Phys. J. C 74 (2014) no.10, 3084 Erratum: [Eur. Phys. J. C 75 (2015) no.11, 535] doi:10.1140/epjc/s10052-015-3754-5, 10.1140/epjc/s10052-014-3084-z [arXiv:1407.8431 [hep-ph]].

[9] F. Caporale, D. Y. Ivanov, B. Murdaca, A. Papa and A. Perri, "The next-to-leading order jet vertex for Mueller-Navelet and forward jets revisited," JHEP 1202 (2012) 101 doi:10.1007/JHEP02(2012)101 [arXiv:1112.3752 [hep-ph]]. 